\title{
Patrons, Authors and Workshops. Books and Book Production in Paris around 1400, edited by Godfried Croenen and Peter Ainsworth
}

\section{Maria Colombo Timelli}

\section{(2) OpenEdition Journals \\ Édition électronique \\ URL : http://journals.openedition.org/studifrancesi/9440 \\ DOI : 10.4000/studifrancesi.9440 \\ ISSN : 2421-5856 \\ Éditeur \\ Rosenberg \& Sellier}

\section{Édition imprimée}

Date de publication : 1 décembre 2007

Pagination : 632-634

ISSN : 0039-2944

\section{Référence électronique}

Maria Colombo Timelli, « Patrons, Authors and Workshops. Books and Book Production in Paris around 1400, edited by Godfried Croenen and Peter Ainsworth », Studi Francesi [En ligne], 153 (LI | III) | 2007, mis en ligne le 30 novembre 2015, consulté le 12 janvier 2021. URL : http://journals.openedition.org/ studifrancesi/9440; DOI : https://doi.org/10.4000/studifrancesi.9440

Ce document a été généré automatiquement le 12 janvier 2021.

\section{c) (†)}

Studi Francesi è distribuita con Licenza Creative Commons Attribuzione - Non commerciale - Non opere derivate 4.0 Internazionale. 


\title{
Patrons, Authors and Workshops. Books and Book Production in Paris around 1400, edited by Godfried Croenen and Peter Ainsworth
}

\author{
Maria Colombo Timelli
}

\section{RÉFÉRENCE}

Patrons, Authors and Workshops. Books and Book Production in Paris around 1400, edited by Godfried CROENEN and Peter AINSWORTH, Louvain, Paris, Dudley MA, Peeters, 2006.

1 Le sujet de ce beau livre exige une approche interdisciplinaire, comme le souligne Godfried Croenen dans ses pages introductives: ainsi, malgré la division des matières que propose la table (I. «Librairies and Commercial Book Production», II. «Bibliophiles: their Collections and their Commissions», III. «Authors and Texts», IV. «Scribes», V. «Artists and Illuminators»), toutes les contributions réunies ici prennent en compte nécessairement plusieurs facettes de la question générale, soit la production du livre sous tous ces aspects, matériels et intellectuels.

2 Kouky FIANU (Métiers et espace: topographie de la fabrication et du commerce du livre à Paris XIII-XV siècle, pp. 21-45) analyse la localisation des différents métiers du livre (copistes, relieurs, enlumineurs, parcheminiers, papetiers, libraires) et étudie son évolution du $\mathrm{XIII}$ au XV $\mathrm{XV}^{\mathrm{e}}$ siècle en rapport avec les changements survenus dans la clientèle.

3 Richard H. ROUSE (Pierre le Portier and the Makers of the Antiphonals of Saint-Jacques, pp. 47-67) reconstruit la figure de Pierre le Portier, libraire et collaborateur de l'ordre de Saint-Jacques-aux-Pèlerins, chargé, entre autres, de la production des antiphonaires; cette enquête permet aussi de jeter quelque lumière sur le coût des livres à Paris au début du Xv siècle. 
Grâce à ses recherches aux Archives Nationales, Mary A. RousE (Archives in the Service of Manuscript Study: the Well-Known Nicolas Flamel, pp. 69-89) montre que la célébrité de Nicolas Flamel comme 'libraire' est mal-fondée, et que ce riche bourgeois de la fin du XIV ${ }^{e}$ siècle n'eut rien à voir avec le monde parisien du livre.

Hilary MADDOCKS (The Rapondi, the Volto Santo di Lucca, and Manuscript Illumination in Paris ca. 1400, pp. 91-122) rappelle le rôle du marchand lucquois Jacques Rapondi, libraire de Philippe le Hardi, due de Bourgogne. Elle étudie en particulier les manuscrits de la Légende du Saint-Voult et leurs illustrations, pour passer ensuite à l'insertion de ce texte, relativement long et surtout strictement rattaché à la ville italienne, dans la version de la Légende dorée amplifiée par l'adjonction des Festes nouvelles.

6 Stéphanie ÖHLUND-RAMBAUD (L'atelier de Jean Trepperel, imprimeur-libraire parisien, 1492-1511, pp. 123-141) reconnaît un 'style Trepperel' dans les bois gravés illustrant ses nombreuses éditions: tant des images «laïques» (surtout pour les romans de chevalerie) que des images à sujet religieux; ce sont essentiellement quelques détails dans le visage des personnages qui contribuent à donner un certain «air de famille» aux différents bois. Selon l'hypothèse de S. O.-R., un nombre important de ces bois auraient été gravés au cours de l'année 1500 .

7 Traducteur du latin et collectionneur de manuscrits, personnage injustement méconnu du premier Humanisme français, Jean Lebègue a droit à deux articles. Dans le premier, Gilbert ouY (Jean Lebègue 1368-1457, auteur, copiste et bibliophile, pp. 143-171) fait le point sur sa biographie et surtout sur sa bibliothèque (avec, en appendice, les notices des manuscrits qui lui ont appartenu). Dans le second, Anne D. HEDEMAN (Making the Fast Present. Visual Translation in Jean Lebègue's 'Twin' manuscripts of Sallust, pp. 173-196) s'attache aux instructions détaillées qu'il a fournies aux artistes chargés d'illustrer son manuscrit personnel de Salluste (Paris, BnF, lat. 5762): Lebègue utilise les images pour y donner une sorte de traduction iconographique du texte latin, et en même temps une adaptation de l'histoire ancienne à l'actualité politique de la France du début du $\mathrm{xv}^{\mathrm{e}}$ siècle.

8 John LOWDEN consacre son article (Beauty or Truth? Making a 'Bible moralisée' in Paris around 1400, pp. 197-222) au ms. Paris, BnF, fr. 166, dont il souligne l'intérêt en prenant en compte trois questions fondamentales: le commanditaire, le ou les auteurs, l'atelier' où il a été réalisé aux alentours de 1400 .

9 Janet F. VAN DER MeUlen (Simon de Lille et sa commande du 'Parfait du Paon'. Pour en finir avec le 'Roman de Perceforest', pp. 223-238) propose de lire le Parfait du Paon, qui conclut le 'cycle' ouvert par les Vœux du Paon et par le Restor du Paon, d'abord commine une réaction au succès de Perceforest: à la demande de Simon de Lille, Jean de le Mote aurait composé hâtivement son poème - où il annule toute possibilité d'attribuer une ascendance glorieuse à la monarchie anglaise - pendant les premiers mois de 1340, en réponse aux provocations de la part d'Edouard III d'Angleterre envers Philippe VI Valois.

Silvère MENEGALDO (Les relations entre poète et mécène dans la 'Prison amoureuse' de Jean Froissart, pp. 239-254) relit la Prison amoureuse comme un témoignage significatif des relations qui s'instaurent au XIV ${ }^{\mathrm{e}}$ siècle entre poète et mécène, dans ce cas entre Froissart et Wenceslas de Brabant: l'inégalité initiale est dépassée en effet grâce à une même relation à l'amour et à la dame. 
11 Dans le but d'identifier le manuscrit de base pour une nouvelle édition des Chroniques de Froissart, Alberto varvaro (Problèmes philologiques du livre IV des 'Chroniques' de Jean Froissart, pp. 255-277) examine en profondeur les variantes du cinquième chapitre du Livre IV; il en arrive ainsi à reconnaître au moins provisoirement la valeur du ms. Bruxelles, KBR, IV.467 (daté 1470): bien qu'il s'agisse d'un manuscrit d'apparence modeste, il fournit un texte complet, correct et très fiable.

Susanne RöHL (Le 'Livre de Mandeville' à Paris autour de 1400, pp. 279-295) souligne l'intérêt des huit manuscrits 'parisiens' (sur une soixantaine en français) du Livre de Mandeville et en décrit les caractères fondamentaux. Compilation d'un grand nombre de sources diverses, à mi-chemin entre œuvre littéraire et texte encyclopédique, il fut très populaire aux XIV e et $\mathrm{Xv}^{\mathrm{e}}$ siècles et servit de source, entre autres, à Jean d'Arras, Christine de Pizan, Philippe de Mézières.

James LAIDLAW (Christine de Pizan: the Making of the Queen's Manuscript - London, British Library, Harley 4431, pp. 297-310) illustre le projet en cours sur le manuscrit que Christine de Pizan offrit à la reine Isabeau en 1414, en soulignant son intérêt tant pour l'éclairage qu'il peut donner sur le fonctionnement de l'atelier de Christine, que pour les informations qu'il offre pour notre connaissance du moyen français.

14 L'article de Margaret CONNolly et Yolanda PLumLey (Crossing the Channel: John Shirley and the Circulation of French Lyric Poetry in England in the early Fifteenth Century, pp. 311-332) concerne un scribe anglais, copiste de Chaucer, Lydgate et d'une anthologie de poésie lyrique (ca. 1430-32) qui contient entre autres trente-et-un poèmes français (liste en appendice II), pour la plupart des ballades, dont les auteurs sont parfois célèbres: Eustache Deschamps, Christine de Pizan, Alain Chartier. Bien que son modèle ne soit pas connu, ce recueil constitue un témoignage remarquable de la circulation de la poésie française au-delà de la Manche.

15 Emilie COTTEREAU (Les copistes en France du Nord autour de 1400: un monde aux multiples visages, pp. 333-354) présente les résultats partiels d'une enquête en cours sur les manuscrits produits à Paris et dans la France septentrionale entre 1370 et 1430 (327 mss. datés et signés). Sont pris en compte: le statut social du copiste, les circonstances de la copie, la langue et le genre du texte, le type de manuscrit.

16 Maria KALATZI (Georgios Hermonymos: a Greek Scribe and Teacher in Paris, pp. 355-365) parcourt la biographie et la carrière de G.H., installé à Paris de 1476 à sa mort (entre 1508 et 1516), où il enseigne le grec et copie plus de cinquante manuscrits, pour la plupart destinés à ses élèves, parmi lesquels on remarque le nom de Lefèvre d'Etaples.

Sue Hellen новввоок (The Properties of Things and Textual Power: Illustrating the French Translation of 'De proprietatibus rerum' and a Latin Precursor, pp. 367-403) s'interroge sur l'illustration du Livre des proprietés des choses (traduction de Jean Corbechon, 1372) dans deux manuscrits (Londres, BL Add 11612, et New York, PML M 537) en les comparant avec celle du ms. Cambridge, Fitzwilliam Museum 15, qui contient le texte latin. Elle montre comment l'iconographie participe, sur le même plan que la traduction, de la volonté de divulgation du savoir et d'implication des lecteurs.

Heidrun ost (Illuminating the 'Roman de la Rose' in the Time of the Debate: The Manuscript of Valencia, pp. 405-435) analyse une sélection d'enluminures du ms. 387 de la Biblioteca Histórica de la Universitad de Valencia, commandité probablement par Philippe le Hardi. Exécutée au tout début $d u x^{e}$ siècle, l'illustration de ce manuscrit ne porte 
cependant aucune trace du débat sur le Roman de la Rose, qui demeura évidemment une querelle purement littéraire.

L'étude de Catherine REYNOLDS (The Workshop of the Master of the Duke of Bedford: Definitions and Identities, pp. 437-472) est fondée sur un manuscrit appartenu à John de Bedford, contenant un Livre d'Heures et un psautier, récemment acquis par la British Library. C.R. discute l'attribution des enluminures et surtout elle souligne l'intérêt des décorations marginales et bordures pour prouver l'existence d'un atelier de facture des manuscrits appartenus au régent d'Angleterre.

Jenny STRATFORD (The Illustration of the 'Songe du Vergier' and some Fifteenth-Century Manuscripts, pp. 473-488): la parenté entre les illustrations des manuscrits appartenus à Charles V (version latine et traduction française du Songe du Vergier) et celles de copies plus tardives, exécutées durant la première moitié $d u \mathrm{xv}^{\mathrm{e}}$ siècle, prouve que les manuscrits de la Tour du Louvre étaient accessibles pendant les dernières années de la guerre de Cent Ans aux partisans du régime anglo-bourguignon; leur dispersion après 1450 est à l'origine d'une tradition iconographique renouvelée.

21 Outre la richesse de l'ensemble des contributions, soulignons pour conclure le nombre et la qualité des reproductions photographiques (82 en noir et blanc et 17 planches en couleur) et la présence de deux précieux indices: noms et sujets (pp. 491-519) et manuscrits cités (pp. 521-529). 